\title{
Prognostic significance of immunohistochemical epithelial-mesenchymal transition markers in skin melanoma patients
}

\begin{abstract}
Aim: To investigate secreted protein acidic and rich in cystein (SPARC) and neural cadherin (NCAD), which are associated with epithelial-mesenchymal transition in primary skin melanoma and nodal metastases and their prognostic impact in melanoma patients. Methods: Expression of proteins was assessed by immunochemistry in archival paraffin samples from 103 primary melanoma tumors and 16 nodal metastases. Results: Increased expression of SPARC and NCAD in primary skin melanoma was associated with decreased overall survival, adverse clinicopathological features and particularly with microsatellitosis (SPARC) and ulceration (NCAD). In univariate Cox regression analysis, both biomarkers were significantly associated with the risk of death; the multivariate Cox regression analysis identified no significance. Conclusion: The most important result of our study was that we confirmed the strict correlation between SPARC and NCAD expression and clinicopathological parameters related with melanoma progression, which is a specific clinical equivalent of the molecular mechanisms of epithelial-mesenchymal transition process and confirms its key role in the disease outcome.
\end{abstract}

First draft submitted: 15 May 2016; Accepted for publication: 22 July 2016; Published online: 2 September 2016

Keywords: biomarker $\bullet$ epithelial-mesenchymal transition $\bullet$ immunohistochemistry - melanoma $\bullet$ microsatellitosis $\bullet \mathrm{N}$-cadherin $\bullet$ prognosis $\bullet \mathrm{SPARC} \bullet$ ulceration

Melanomas are one of the most aggressive malignant neoplasms and their clinical course is often unpredictable. Considering the availability of systemic and targeted therapy options, it is of key importance to understand complex molecular mechanisms that regulate melanomagenesis and promote the spread of cancer cells. One of the hypotheses is concerned with epithelial-mesenchymal transition (EMT). It was assumed that the process involves epithelial phenotype cells losing their intercellular cohesion and gaining migratory capacity and thus being able to form nodal and distant metastases [1]. Even though skin melanomas are not epithelial in origin, their cells initially have a set of features that is characteristic of epithelial phenotype and acquire properties charac- teristic of mesenchymal phenotype as their metastatic potential increases [2]. EMT is initiated by complex interactions between the microenvironment and melanocytes. Intracellular signaling pathways are triggered that induce changes in the expression of several proteins $[1,3]$. The proteins are both mediators and specific markers of EMT and include SPARC - extracellular matrix (ECM) protein and adhesion protein - neural cadherin (NCAD) [1,4] .

Secreted protein acidic and rich in cystein (SPARC; osteonectin, BM40) [5] belongs to a group of extracellular noncollagen glycoproteins that bind $\mathrm{Ca}^{2+}$. Its role in the biology of malignant neoplasms is complex and pleiotropic. It was shown that increased activity of SPARC may both suppress the development of
Malgorzata Pieniazek ${ }^{*, 1}$, Piotr Donizy², Agnieszka Halon', Marek Leskiewicz ${ }^{3}$ \& Rafal Matkowski ${ }^{4,5}$

'Department of Clinical Oncology، Tadeusz Koszarowski Regional Oncology Center, Opole, Katowicka 66a, Poland ${ }^{2}$ Department of Pathomorphology \& Oncological Cytology, Wroclaw Medical University, Borowska 213, 50-556 Wroclaw, Poland ${ }^{3}$ Department of Statistics, Wroclaw University of Economics, Komandorska 118-120, 53-345 Wroclaw, Poland ${ }^{4}$ Department of Oncology \& Division of Surgical Oncology, Wroclaw Medical University, pl. Hirszfelda 12, 53-413 Wroclaw, Poland ${ }^{5}$ Lower Silesian Oncology Centre, pl. Hirszfelda 12, 53-413 Wroclaw, Poland *Author for correspondence: malgorzatapieniazek@interia.pl
Future 
cancer (e.g., neuroblastoma, ovarian cancer, myelodysplastic syndromes and acute myeloid leukemia [6]) and be a malignancy marker (e.g., breast cancer, lung cancer, prostate cancer, bladder cancer, gastrointestinal cancers, gliomas and skin melanomas [6,7]). SPARC contributes to cancer progression both as regards ECM environment and cytobiochemical processes within a cell. In the microenvironment it promotes neoplastic infiltration and metastasis formation mostly through remodeling. SPARC enhances the activity of MMP-2 and MMP-9 [8] that mainly degrade collagen type IV, a major component of the basement membrane. The structural changes are also an element of angiogenesis, and the cleavage of SPARC by MMP-3 generates proangiogenic peptides which contain GHK sequence (N-glycyl-Lhistidyl-L-lysine-OH) [9]. Interestingly, high concentration of SPARC in ECM stimulates cancer progression as it helps cancer cells evade immune surveillance [10]. SPARC also enables cancer cells to acquire invasive phenotype $[6,11]$ by deregulating adhesion complexes, reorganizing actin stress fibers and partly through indirect interactions with integrin-linked kinase. SPARC affects intracellular processes through interaction with various growth factors, for example, TGF- $\beta$, which is the main regulator of EMT process [11]. Findings from many studies on the role of SPARC in malignant tumor growth suggest that it is one of the key elements involved in cancer progression and metastasis formation.

Neural cadherin, in other words, NCAD, also called cadherin-2 or cluster of differentiation (CD325) is one of classic type I cadherins, adhesion protein superfamily that mediates intercellular interactions. The cells with surface expression of NCAD are spindle-shaped, apolar and have enhanced migratory capacity. It was demonstrated that NCAD expression induces cell migration in the following cancers: breast cancer [12], bladder cancer [13,14], prostate cancer [15], esophageal squamous cell carcinoma [16] and skin melanomas $[2,11]$. The acquisition of expression or overexpression of NCAD by cancer cells with simultaneous decrease or maintenance of ECAD expression (epithelial cadherin, characteristic of epithelial phenotype) was termed as cadherin switching or EN switch [17]. It is one of the first elements of EMT responsible for structural and functional changes of the specific population of cancer cells that enhance their motility, ability to penetrate the basement membrane and the ECM, as well as distant migration capacity [17]. It was shown that in carcinogenesis NCAD affects not only cell phenotype, but NCAD can also be an effector of tyrosine kinase receptors, for example, FGFR [18-21] or PDGFR [22], which are strictly related with the major metabolic pathways of EMT $[1,4]$. NCAD also regulates Rho family GTPases (RhoA/B/C, Rac1 and Cdc42) which are involved in cell motility - formation of filopodia (e.g., Cdc42), lamellipodia (e.g., Rac1) and actin stress fibers (e.g., RhoA) [4,23]. Additionally, it was postulated that NCAD occurring on endothelial cells may interact with cancer cells' NCAD and thus facilitate their adhesion and then vascular invasion [24]. The outlined studies and correlations suggest that SPARC and NCAD play a critical role in tumor growth and neoplasm progression.

The aim of the study was to examine the correlation between the expression of SPARC and NCAD and the main clinicopathological parameters, and to determine their prognostic significance.

Most of the studies into NCAD overexpression were focused on the reduced expression of ECAD which is why the comparison of NCAD and SPARC seems particularly interesting. Our literature search showed that the interplay between SPARC and NCAD was studied only as one of the elements of EMT molecular pathways. The correlations between the two biomarkers and clinicopathological parameters demonstrated in the paper are a specific clinical equivalent of the molecular mechanisms of EMT process and confirm the key role of EMT in the disease outcome. Our study provides new insights into the prognostic significance of the two proteins and contributes to the current knowledge of their correlation with clinicopathological parameters.

\section{Methods}

\section{Patients \& ethics statements}

The study group consisted of 103 patients with cutaneous malignant melanoma who were diagnosed between 2005 and 2010 and treated in the Lower Silesian Oncology Center in Wroclaw, Poland. Additionally, tissue material obtained from 16 nodal metastatic foci from the same patients was included in the study. The only inclusion criteria were the availability of appropriate quality paraffin blocks - the study was performed using complete cross section of the tumor (and not microarrays) and the availability of medical documentation. No additional exclusion criteria were applied. Comprehensive clinical data were obtained from archival medical records. The diagnostic and therapeutic procedures utilized were determined from medical records in the Oncology Outpatient Clinic of the Lower Silesian Oncology Center and data provided by the Lower Silesian Cancer Registry and Civil Register Office in Wroclaw, Poland. The retrospective study was approved by the Ethical Committee of the Wroclaw Medical University (Wroclaw, Poland; number 376/2010).

The clinicopathological profile of patients is shown in Table 1. The median follow-up time was 3 years and 7 months and the number of events was 31 . 
Tumor samples \& histopathological evaluation Tumor specimens were fixed in $10 \%$ natural-buffered formalin and embedded in paraffin. All hematoxylin and eosin stained sections were examined by two pathologists. They verified the diagnosis and studied the parameters of the primary tumor listed in Table 2.

\section{Immunohistochemistry}

Formalin-fixed, paraffin embedded tissue was freshly cut $(4 \mu \mathrm{m})$. The sections were mounted on superfrost slides (Menzel Glaser, Brunswick, Germany), dewaxed with xylene and gradually hydrated. The activity of endogenous peroxidase was blocked by 5 -min exposure to $3 \% \mathrm{H}_{2} \mathrm{O}_{2}$. The sections were boiled for $15 \mathrm{~min}$ at 250W in Antigen Retrieval Solution (DakoCytomation, Glostrup, Denmark). Immunohistochemical reactions were then performed using monoclonal antibodies detecting SPARC (clone PP16; Santa Cruz Biotechnology, TX, USA) and NCAD (clone D4; Santa Cruz Biotechnology). The tested sections were incubated with antibodies for $1 \mathrm{~h}$ at room temperature. The subsequent incubations involved biotinylated antibodies (15 min at room temperature) and streptavidin-biotinylated peroxidase complex (15 $\mathrm{min}$ at room temperature; LSAB+, HRP [DakoCytomation]). DAB (Vector Laboratories, Peterborough, UK) was used as a chromogen (10 $\mathrm{min}$ at room temperature). All sections were counterstained with Meyer's hematoxylin. In every case control reactions were performed, with the relevant antibody substituted by Primary Mouse Negative Control (DakoCytomation).

\section{Evaluation of reaction intensity}

The intensity of the immunohistochemical reaction was estimated independently by two pathologists. Doubtful cases were re-evaluated under a doubleheaded microscope and staining was discussed until consensus was achieved. The expression of these proteins was calculated using a semi-quantitative method by two pathologists. Two immunohistochemical reaction parameters were considered when evaluating the expression of the foregoing proteins: the percentage of cells with a positive cytoplasmic reaction (the percentage of reactive tissue) and the reaction intensity. The final immunohistochemical reaction results are expressed according to the semi-quantitative IRS (ImmunoReactive Score) scale devised by Remmele and Stenger [25]. This scale assigns a score for the percentage of cells demonstrating reaction ( $0-4$ points) and for reaction intensity ( $0-3$ points). The final result is the product of the scores for these two parameters ( $0-12$ points) and is referred to as an IRS factor or score.
Table 1. Clinicopathological characteristics of the patients. Clinicopathological characteristics Number (\%)

All patients $103(100.0)$

Mean age (years): $56.34 \pm 15.37$; median (years): 58 Sex:

- Female

- Male $43(41.7)$

Tumor site:

- Head/neck

$15(14.6)$

- Upper and lower limbs $\quad 45$ (43.7)

- Trunk 41 (39.8)

- Hand/foot 2 (1.9)

Primary tumor (pT):

- pT1 $34(33.0)$

- pT2 $21(20.4)$

- pT3 25 (24.3)

- pT4 $23(22.3)$

SLNB status: $\quad 60(57.7)$

- No metastases (SNLB-) $\quad 48(81.4)$

- Metastases present (SNLB+) 11 (18.6)

Regional nodal metastases $(\mathrm{pN})$ :

- No metastasis ( $\mathrm{pN}-$ ) $\quad 87(84.5)$

- Metastases present $(\mathrm{pN}+) \quad 16$ (15.5)

Recurrence:

- No $86(84.5)$

- Yes 17 (16.5)

Distant metastases:

- No $\quad 98$ (95.1)

- Yes $5(4.9)$

SLNB: Sentinel lymph node biopsy.

\section{Statistical analysis}

Statistical analysis was performed using the Statistica 10.0 and IBM SPSS 21 software packages. Overall survival (OS) was defined as the time between the primary surgical treatment and death, and OS was censored at last follow-up for patients who were still alive. Cancer-specific OS (CSOS) was defined as the time between the primary surgical treatment and cancerassociated death, and was censored at the last follow-up for surviving patients.

The Spearman rank correlation was used to analyze associations between mitotic rate and the presence of ulceration and clinicopathological parameters. Differences between the means were tested with a nonparametric test (Mann-Whitney U test and Kruskal-Wal- 


\begin{tabular}{|c|c|}
\hline $\begin{array}{l}\text { Histopathological parameters } \\
\text { of primary tumors }\end{array}$ & Number (\%) \\
\hline \multicolumn{2}{|l|}{ Breslow thickness: } \\
\hline$-<1 \mathrm{~mm}$ & $34(33.01)$ \\
\hline$-1.01-2.00 \mathrm{~mm}$ & $20(19.42)$ \\
\hline$-2.01-4.00 \mathrm{~mm}$ & $26(25.24)$ \\
\hline$->4 \mathrm{~mm}$ & $23(22.33)$ \\
\hline \multicolumn{2}{|l|}{ Clark level: } \\
\hline-1 & $0(0.0)$ \\
\hline-11 & $18(17.48)$ \\
\hline$-1 \mathrm{II}$ & $49(47.57)$ \\
\hline$-I V$ & $25(24.27)$ \\
\hline$-V$ & $11(10.68)$ \\
\hline \multicolumn{2}{|l|}{ Histologic type: } \\
\hline- SSM & $68(66.02)$ \\
\hline - NMM & $31(30.1)$ \\
\hline - ALM & $4(3.88)$ \\
\hline \multicolumn{2}{|l|}{ Mitotic rate: } \\
\hline-0 & 45 (43.69) \\
\hline$-1-2$ & $26(25.24)$ \\
\hline$-\geq 3$ & $32(31.07)$ \\
\hline \multicolumn{2}{|l|}{ Ulceration: } \\
\hline$-\mathrm{No}$ & $55(53.4)$ \\
\hline - Yes & $48(46.6)$ \\
\hline \multicolumn{2}{|l|}{ Lymphangioinvasion: } \\
\hline$-\mathrm{No}$ & $73(7087)$ \\
\hline- Yes & $30(29.13)$ \\
\hline \multicolumn{2}{|l|}{ Growth phase: } \\
\hline - Radial & $3(2.91)$ \\
\hline - Vertical & $100(97.09)$ \\
\hline \multicolumn{2}{|l|}{ TILs: } \\
\hline$-\mathrm{No}$ & $18(17.48)$ \\
\hline - Nonbrisk & $33(32.04)$ \\
\hline - Brisk & $52(50.48)$ \\
\hline \multicolumn{2}{|l|}{ Microsatellitosis: } \\
\hline- No & $97(94.17)$ \\
\hline - Yes & $6(5.83)$ \\
\hline \multicolumn{2}{|l|}{ Tumor regression: } \\
\hline$-\mathrm{No}$ & $95(92.23)$ \\
\hline - Yes & $8(7.77)$ \\
\hline
\end{tabular}

lis test), the log-rank test was used to compare survival in two groups with either low or high expression of NCAD or SPARC, the OS rate was estimated by the Kaplan-Meier method and the influence of explanatory variables on death risk was analyzed by means of the Cox proportional hazard regression. p-values $<0.05$ were considered statistically significant.

\section{Results}

\section{SPARC \& NCAD expression in primary}

\section{\& metastatic tumors}

SPARC and NCAD expression was observed only in melanoma cells both in tissue material obtained from the primary tumor and nodal metastatic foci. No SPARC or NCAD immunoreactivity was identified in stromal compartment of tumor or lymphocytes from regional lymph nodes. Cancer cells of the primary tumor and metastatic cells were found to exhibit only diffuse cytoplasmic expression (Figure 1).

SPARC expression (IRS $>0$ ) was observed in 80 primary tumors $(80 / 103,78 \%)$. The mean IRS for SPARC in primary tumors was 3.72 (standard deviation [SD]: 3.142). In nodal metastatic foci the protein was expressed in 9 of 16 cases (56\%). The mean IRS for metastatic lesions was 4.25 (SD 4.60).

NCAD expression (IRS $>0$ ) was reported in melanoma cells of 81 primary tumors $(81 / 103,79 \%)$. The mean IRS for NCAD in primary tumors was 4.22 (SD: 3.181). Also in the metastatic lesions under study the majority of cells showed expression of NCAD. NCAD immunoreactivity was observed in 11 of 16 (69\%) metastatic foci. The mean IRS for NCAD in metastatic lesions was 4.38 (SD: 4.30).

\section{Correlations between SPARC \& NCAD}

expression in primary tumor \& metastatic foci \& histopathological \& clinicopathological parameters

The two EMT markers were associated with all histopathological and clinicopathological parameters. The results are shown in Tables 3 \& 4 .

\section{The effect of SPARC \& NCAD expression on} 5-year survival in skin melanoma patients Kaplan-Meier estimators for OS \& CSOS

It was demonstrated that even minor expression of SPARC (IRS $=3.4$ ) is associated with shorter OS ( $\mathrm{p}=0.015$ and $\mathrm{p}=0.007$, respectively). However, the most critical factor affecting survival in skin melanoma patients is SPARC expression defined as IRS $\geq 6$ $(p=0.003)$ (Figure 2 ). Intensity of reaction and the percentage of SPARC-positive cells also have an impact on survival. Similarly, the impact of SPARC expression in primary skin melanomas on CSOS was exam- 


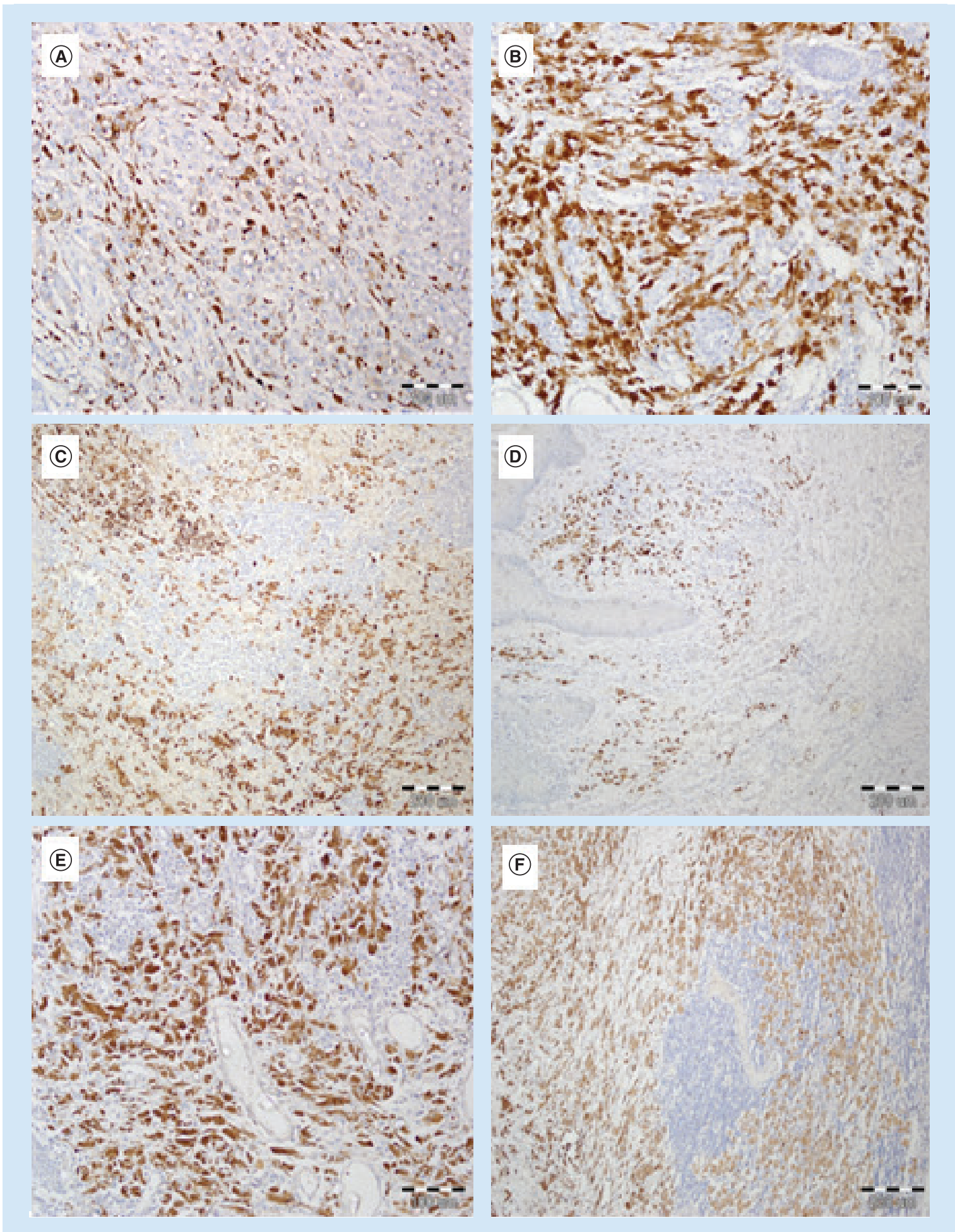

Figure 1. Immunohistochemically visualized expression of secreted protein acidic and rich in cystein and neural cadherin in cutaneous melanoma. Low cytoplasmic expression of SPARC in melanoma cells (A), 200x, hematoxylin. Diffuse and strong cytoplasmic reactivity of secreted protein acidic and rich in cystein (SPARC) in neoplastic melanocytes (B), 200x, hematoxylin. SPARC expression in nodal metastases of melanoma-enhanced reactivity in neoplastic melanoma cells and lack of expression in normal lymphocytes (C), 100x, hematoxylin. Low cytoplasmic expression of NCAD in melanoma cells (D), 100x, hematoxylin. Elevated cytoplasmic immunoreactivity of neural cadherin (NCAD) in melanoma cells (E), 200x, hematoxylin. Diffuse and strong expression of NCAD in neoplastic melanocytes in nodal metastasis (F), 100x, hematoxylin. 


\begin{tabular}{|c|c|c|c|c|c|c|}
\hline \multirow{2}{*}{$\begin{array}{l}\text { Clinicopathological } \\
\text { parameters }\end{array}$} & \multicolumn{3}{|c|}{ SPARC expression - primary tumor } & \multicolumn{3}{|c|}{ SPARC expression - nodal metastasis } \\
\hline & $\%^{\dagger}$ & $\operatorname{lnt}^{\ddagger}$ & IRS $§$ & $\%^{+}$ & $\operatorname{lnt}^{*}$ & IRS $\$$ \\
\hline $\mathrm{pT}^{\uparrow}$ & $0.039 *$ & $0.039 *$ & $0.015^{*}$ & $0.101 *$ & $0.049 *$ & 0.085 \\
\hline $\mathrm{pN}^{\#}$ & 0.188 & 0.309 & 0.153 & 0.425 & 0.447 & 0.395 \\
\hline Distant metastases ${ }^{\#}$ & 0.128 & 0.187 & 0.054 & 0.933 & 0.932 & 0.934 \\
\hline Recurrence $\#$ & 0.054 & 0.169 & $0.032 *$ & 0.344 & 0.189 & 0.293 \\
\hline Age $e^{\pi}$ & 0.342 & 0.468 & 0.414 & $0.025^{*}$ & $0.048 *$ & $0.040 *$ \\
\hline Gender ${ }^{\#}$ & 0.114 & 0.162 & 0.196 & 0.656 & 0.424 & 0.580 \\
\hline 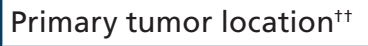 & 0.289 & 0.266 & 0.478 & 0.620 & 0.321 & 0.469 \\
\hline Breslow thickness" & $0.026 *$ & $0.016 *$ & $0.006^{*}$ & 0.121 & 0.086 & 0.128 \\
\hline Clark levelø & 0.017 & $<0.0001 *$ & $0.001 *$ & 0.351 & 0.498 & 0.398 \\
\hline Growth phase & 0.368 & 0.202 & 0.27 & \multicolumn{3}{|c|}{$100 \%$ vertical growth phase } \\
\hline Histologic type $^{\dagger \dagger}$ & 0.87 & 0.414 & 0.615 & 0.095 & 0.06 & 0.108 \\
\hline Mitotic rateף & 0.054 & $0.033^{*}$ & $0.033^{*}$ & 0.069 & $0.013^{*}$ & $0.038^{*}$ \\
\hline Ulceration ${ }^{\#}$ & 0.219 & 0.063 & 0.063 & 0.702 & 0.433 & 0.612 \\
\hline Lymphangioinvasion" ${ }^{\#}$ & 0.056 & 0.97 & $0.019 *$ & 0.362 & 0.242 & 0.256 \\
\hline Microsatellitosis" & 0.254 & $0.026^{*}$ & 0.077 & 0.305 & 0.293 & 0.307 \\
\hline $\begin{array}{l}\text { Tumor-infiltrating } \\
\text { lymphocytes }{ }^{\dagger+}\end{array}$ & 0.557 & 0.739 & 0.697 & 0.53 & 0.273 & 0.428 \\
\hline Tumor regression" & 0.063 & 0.155 & 0.099 & \multicolumn{3}{|c|}{$100 \%$ without regression } \\
\hline \multicolumn{7}{|c|}{$\begin{array}{l}\text { *Statistically significant results }(p<0.05) \text {. } \\
\text { "Percentage of SPARC-positive melanoma cells }(0-4 \text { points). } \\
\text { "Intensity of immunohistochemical reaction }(0-3 \text { points). } \\
\text { \$ImmunoReactive Score (product of the scores for the percentage of positive cells and intensity of reaction [0-12 point]). } \\
\text { "p-value of Spearman rank correlation. } \\
{ }^{*} p \text {-value of Mann-Whitney U test. } \\
+{ }^{+p} p \text {-value of Kruskal-Wallis test. } \\
p N \text { : Histopathologically proven regional nodal metastasis; } \mathrm{pT} \text { : Histopathologically proven size of the primary tumor; SPARC: Secreted protein } \\
\text { acidic and rich in cystein. }\end{array}$} \\
\hline
\end{tabular}

ined. None of the results was statistically significant $(\mathrm{p}>0.05)$.

Analyses of OS associated with NCAD expression in primary tumor cells were performed for all immunoreactivity subgroups. It was shown that any NCAD expression in melanoma cells results in significantly worse prognosis for patients. Each of the results is statistically significant $(p<0.005)$, and prognostic significance increases with the increase of IRS and is the highest for IRS $\geq 6$ (Figure 2). As it is in the case of SPARC, not only NCAD expression itself expressed as IRS, but also its specific parameters have an impact on prognosis. For NCAD, the presence of any intensity of reaction, even weak, is associated with worse prognosis, in other words, OS shorter by a minimum of $30 \%$. Additionally, the presence of NCAD-positive cells at the level of only $25-50 \%$ is indicative of a poor outcome. Also the estimations for CSOS clearly demonstrate that increased immunoreactivity of NCAD (Figure 2) and its parameters: the presence of NCAD- positive cells and intense color reaction indicate poor prognosis.

Similar OS and CSOS analyses were performed for metastatic lesions. No impact on OS was observed.

Cox univariate \& multivariate analysis of clinicopathological parameters

Cox univariate analysis confirmed that increased immunoreactivity of SPARC and NCAD ( $p=0.0064$ and $\mathrm{p}=0.019)$ has an impact on the risk of death in 5-year follow-up (Table 5), but multivariate analysis did not confirm their status of independent prognostic factors.

\section{Discussion}

The first study into SPARC expression in skin melanoma in correlation with clinical outcome was conducted by Massi et al. [26]. Our work expands the hypotheses put forward by Massi et al. Our results additionally confirm the correlation between SPARC and poor prognosis but on a group that is almost three- 
times bigger, and not only for thin melanomas, as pT1 $(<1 \mathrm{~mm})$ patients constitute only approximately a third of the study group $(33.01 \%)$, but also for a more advanced infiltration. Our findings suggest that SPARC is a marker of poor prognosis at each level of Breslow's depth. What is more, our results add new information to the results obtained by Massi et al. We provide new insights into the correlation between SPARC and clinicopathological factors such as Breslow's depth and Clark's level, pT, mitotic rate, the presence of lymphangio-invasion, microsatellitosis (in the case of SPARC reaction intensity) and the relapse of cutaneous melanomas. What is particularly interesting is that a correlation with microsatellitosis was shown. The literature search did not reveal a similar report.

We think that positive correlation between SPARC overexpression and higher mitotic rate suggests that SPARC is upregulated in the primary tumor cells with high proliferative, invasive and metastatic potential.
That is extremely interesting, since our hypothesis is partly confirmed by Botti et al. who observed that SPARC overexpression in melanoma cells may be involved in tumor spreading to the lung [27]. The analyses showed significant positive correlation between SPARC expression in primary tumor and the respective metastasis $(\mathrm{p}<0.002)$, and high reactivity of SPARC was also observed in metastasis.

It should be noted that SPARC was studied not only in melanoma cells. Ikuta et al. [28] determined the concentration of soluble SPARC in the sera of patients with melanomas, may be a marker for early stage melanomas.

The results of clinical and experimental studies outlined above indicate that SPARC plays a critical role in melanoma growth and greatly contributes to the formation of metastases.

SPARC overexpression in melanocytes results in the decrease of epithelial cadherin level, which

Table 4. Correlations between clinicopathological and histopathological characteristics and neural cadherin expression parameters in primary tumors and nodal metastases.

\begin{tabular}{|c|c|c|c|c|c|c|}
\hline \multirow{2}{*}{$\begin{array}{l}\text { Clinicopathological } \\
\text { parameters }\end{array}$} & \multicolumn{3}{|c|}{ NCAD expression - primary tumor } & \multicolumn{3}{|c|}{ NCAD expression - nodal metastasis } \\
\hline & $\%^{\dagger}$ & $\operatorname{lnt}^{\ddagger}$ & IRS $\S$ & $\%^{+}$ & $\operatorname{lnt}^{\ddagger}$ & IRS $\S$ \\
\hline $\mathrm{pT}^{\text {ๆ }}$ & 0.011 * & $0.002 *$ & $0.009 *$ & 0.093 & 0.112 & 0.101 \\
\hline $\mathrm{pN}^{\#}$ & $0.033 *$ & 0.136 & $0.035^{*}$ & 0.823 & 0.866 & 0.868 \\
\hline Distant metastases ${ }^{\#}$ & 0.060 & 0.058 & $0.008^{*}$ & 0.62 & 0.94 & 1 \\
\hline Recurrence $\#$ & $0.007^{*}$ & 0.063 & $0.003 *$ & 0.785 & 0.412 & 0.666 \\
\hline $\mathrm{Age}^{\pi}$ & 0.487 & 0.124 & 0.347 & 0.050 & $0.008 *$ & $0.018 *$ \\
\hline Gender ${ }^{\#}$ & 0.204 & 0.085 & 0.222 & 0.127 & 0.662 & 0.451 \\
\hline Primary tumor location $^{\dagger+}$ & 252 & 0.849 & 0.297 & 0.645 & 0.205 & 0.36 \\
\hline Breslow thickness" & $0.008^{*}$ & $0.001 *$ & $0.005^{*}$ & 0.188 & 0.155 & 0.16 \\
\hline Clark levelף & $0.008 *$ & $<0.001 *$ & $0.002 *$ & 0.086 & 0.221 & 0.186 \\
\hline Growth phase & $0.045^{*}$ & 0.297 & 0.060 & \multicolumn{3}{|c|}{$100 \%$ vertical growth phase } \\
\hline Histologic type ${ }^{\dagger \dagger}$ & 0.933 & 0.290 & 0.922 & 0.549 & 0.101 & 0.196 \\
\hline Mitotic rate & 0.073 & $0.001 *$ & $0.036^{*}$ & 0.494 & 0.273 & 0.299 \\
\hline Ulceration ${ }^{\#}$ & 0.426 & $0.002 *$ & 0.106 & 0.492 & 0.707 & 0.711 \\
\hline Lymphangioinvasion" & $0.003^{*}$ & $0.038 *$ & $0.003 *$ & 0.180 & 0.654 & 0.377 \\
\hline Microsatellitosis" & 0.790 & 0.253 & 0.424 & 0.219 & 0.217 & 0.224 \\
\hline $\begin{array}{l}\text { Tumor-infiltrating } \\
\text { lymphocytes }^{\dagger+}\end{array}$ & 0.464 & 0.584 & 0.571 & 0.998 & 0.934 & 0.959 \\
\hline Tumor regression\# & 0.065 & 0.463 & 0.198 & $100 \%$ & ut regre & \\
\hline \multicolumn{7}{|c|}{ 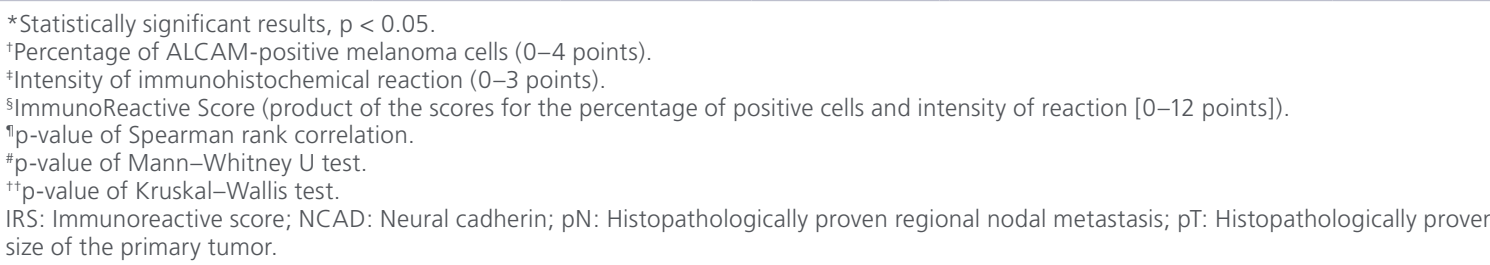 } \\
\hline
\end{tabular}



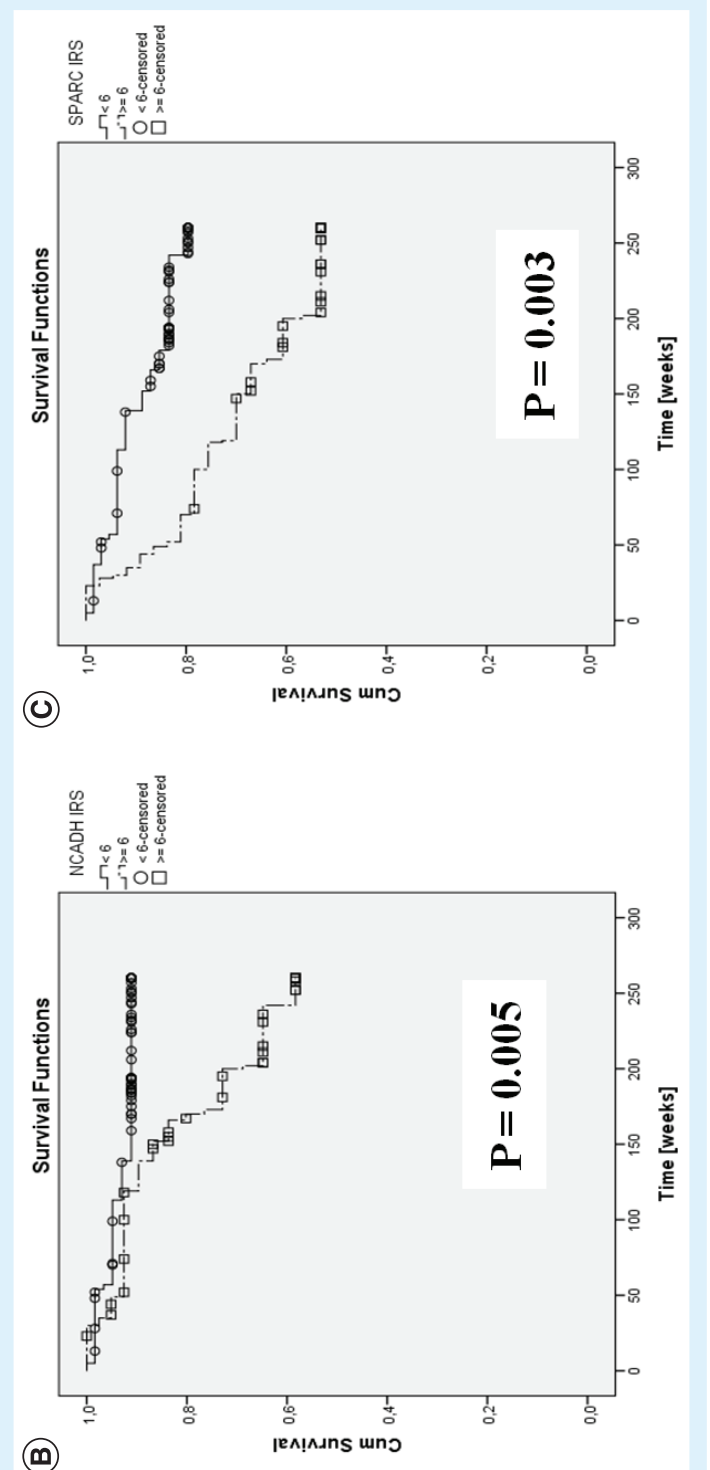

(9)

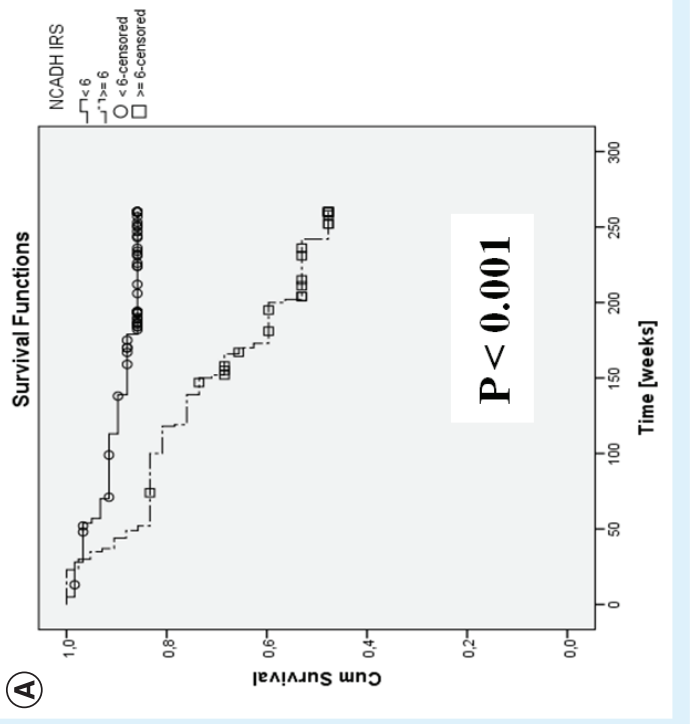

causes melanocytes to be liberated from keratinocyte control and grow to become melanoma cells with migration and invasion capacity characteristic of cancer cell [29]. Experimental studies also showed the correlation between SPARC concentration and metabolic pathways that affect melanoma cells proliferation. It was shown, for example, that suppression of SPARC expression in melanoma cell activates p53-dependent p21 Cip21/Waf1 pathway. Depletion of SPARC stabilizes $\mathrm{p} 53$, and as a result activates the above metabolic pathway and induces G2/M cell cycle arrest [5]. Recent reports indicate that $\mathrm{p} 21^{\mathrm{Cip} 21 / \mathrm{Waf} 1}$ inhibits EMT, decreases cells' migratory potential and eliminates their mesenchymal changes not only in melanoma but also in breast cancer [5]. It was also shown that the absence of intracellular SPARC inhibits melanoma cells growth and their apoptosis with G1 arrest, and thus inhibits tumor growth [7]. Silencing of SPARC expression is accompanied by p21 accumulation, however its role in inhibition of cell cycle remains to be determined [7].

Our study suggests that any NCAD expression in melanoma cells results in significantly worse prognosis for patients and the analyses of the interdependence for NCAD showed statistically significant correlations with clinicopathological parameters identical to those for SPARC. Additionally, positive correlation was observed between increased NCAD reaction intensity and the presence of ulceration. On top of that, positive correlations were noted between high NCAD expression and the presence of regional lymph node metastasis and distant metastasis.

The prognostic significance of NCAD remains unclear in the literature and the findings reported by research teams are contradictory in some aspects. Bachmann et al. did not find any correlation between NCAD expression and the clinical outcome of melanoma [30]. Kreizenbeck et al. showed that increased expression of NCAD is associated with better OS $(p=0.03)$ in Cox univariate analysis, which was not confirmed in multivariate analysis $(\mathrm{p}=0.5)$ [31].

There are few studies focused on the correlation of the NCAD itself with clinicopathological factors. There are reports that confirm the correlation between Breslow's depth and lymph node metastasis that was noted by us. Lade-Keller et al. analyzed 394 melanomas and showed that high expression of NCAD is strictly correlated with Breslow's depth ( $p$ $<0.001$ ) [32], and Alonso et al. proved that NCAD expression is associated with higher incidence of lymph node metastasis ( $p=0.013$ ) [2]. Other reports on increased expression of NCAD are related with EN switch process where NCAD overexpression is strictly related with decreased expression of ECAD. E $\downarrow N \uparrow$ 
profile is strictly related with shorter OS and considerably shorter cancer-specific OS $(\mathrm{p}=0.005)$ and distant metastasis-free survival $(\mathrm{p}=0.01)$. In multivariate analysis it is an independent prognostic factor for them $(\mathrm{p}=0.04$ and $\mathrm{p}=0.02$, respectively) [32]. Therefore, the comparison of NCAD and SPARC seems particularly interesting.

Literature review and our findings unequivocally confirm the correlation between NCAD and melanoma progression. Detailed analysis of the correlation between NCAD and melanoma intracellular processes and their impact on processes related with increased invasion serves as an explanation to the results of clinical analyses. For example, blocking NCAD expression in cancer melanocytes inhibits their transendothelial migration [33]. Additionally, NCAD and NCAD-mediated intercellular interactions affect melanoma cell apoptosis. Overexpression promotes melanoma cell survival by activating the Akt anti-apoptotic metabolic pathway. Blocking NCAD with specific antibodies within the cells results in activating apoptosis pathways [34]. In yet another study, NCAD was shown to affect anoikis which is apoptosis induced when a cell is detached from ECM [35]. NCAD initiates a cascade of metabolic signals which trigger resistance to anoikis [36]. Moreover, NCAD was demonstrated to be involved in angiogenesis. A study using prostate cancer cells demonstrated that blocking NCAD expression is associated with a decrease in angiogenic molecules (e.g., MCP-1) [15]. NCAD regulates the level of proangiogenic factors by promoting Akt phosphorylation, in other words, the metabolic pathway that inhibits anoikis [15]. PI3K/Akt is one of the most important pathways regulating EMT [1,4].

The most important result of our study was that we confirmed the strict correlation between SPARC and NCAD expression and clinicopathological parameters related with melanoma progression, which is a specific clinical equivalent of the molecular mechanisms of EMT process and confirms the key role of EMT in the disease outcome. Our results also contribute to the current knowledge of the clinicopathological correlations and overexpression of the two biomarkers. The correlations between microsatellitosis and SPARC and between ulceration and NCAD seem to be particularly interesting. We did not find a similar report in the available medical literature.

\section{Conclusion}

OS in skin melanoma is worse in individuals with overexpression of SPARC and NCAD in primary tumor cells, while cancer-specific survival is affected only by overexpression of NCAD. SPARC and NCAD inter-

\begin{tabular}{|c|c|c|}
\hline \multirow[t]{2}{*}{ Clinicopathological parameters } & \multicolumn{2}{|c|}{ Univariate models } \\
\hline & p-value & HR \\
\hline Primary tumor $(\mathrm{pT})$ & $<0.001$ & 2.04 \\
\hline Distant metastases & $<0.001$ & 6.98 \\
\hline Breslow thickness (mm) & $<0.001$ & 2,11 \\
\hline SPARC IRS & 0.0042 & 1.17 \\
\hline NCAD IRS & 0.0024 & 1.19 \\
\hline
\end{tabular}

play in skin melanoma progression. EMT transition taking the form of SPARC and NCAD overexpression is correlated with the presence of classic poor prognostic factors. The arguments for it are in particular the correlation with the presence of lymphangio-invasion, high mitotic rate, ulceration (NCAD) and microsatellitosis (SPARC).

\section{Author contributions}

M Pieniazek, P Donizy, A Halon, R Matkowski: study concepts and design; M Pieniazek, P Donizy, R Matkowski: data acquisition; M Pieniazek, P Donizy, R Matkowski, M Leskiewicz, A Halon: data analysis and interpretation; M Leskiewicz: statistical analysis; M Pieniazek, P Donizy, R Matkowski: manuscript preparation and editing. All authors read and approved the final manuscript.

\section{Financial \& competing interests disclosure}

This study was supported by Wroclaw Medical University research grant Pbmn108 and ST-843. The authors have no other relevant affiliations or financial involvement with any organization or entity with a financial interest in or financial conflict with the subject matter or materials discussed in the manuscript apart from those disclosed.

No writing assistance was utilized in the production of this manuscript.

\section{Ethical conduct of research}

The authors state that they have obtained appropriate institutional review board approval or have followed the principles outlined in the Declaration of Helsinki for all human or animal experimental investigations. In addition, for investigations involving human subjects, informed consent has been obtained from the participants involved.

\section{Open access}

This work is licensed under the Creative Commons Attribution 4.0 License. To view a copy of this license, visit http://creativecommons.org/licenses/by/4.0/ 
Executive summary

- Epithelial-mesenchymal transition taking the form of SPARC and NCAD overexpression is correlated with the presence of classic poor prognostic factors: the presence of lymphangio-invasion, high mitotic rate, ulceration (NCAD) and microsatellitosis (SPARC). SPARC and NCAD interplay in cutaneous melanoma progression.

- Increased SPARC expression (IRS $\geq 6$ ) in primary tumor cells of cutaneous melanoma results in shorter $(p=0.003)$ overall survival. Poor prognosis is also determined by the parameters of the protein expression high intensity of SPARC $(p=0.001)$ and the percentage of SPARC-positive cells.

- Increased immunoexpression of SPARC is positively correlated with more advanced melanomas in Breslow and Clark scale $(p=0.006$ and $p=0.001)$, higher mitotic rate $(p=0.033)$ and the presence of lymphangio-invasion $(p=0.019)$ and microsatellitosis ( $p=0.026$ for expression intensity).

- Strong SPARC expression is correlated with a more advanced $\mathrm{pT}(\mathrm{p}=0.015)$, and cutaneous melanoma relapse $(p=0.032)$.

- Overexpression of NCAD (IRS $\geq 6)$ in primary tumor cells reduces overall survival by almost $50 \%(p<0.001)$. The presence of NCAD-positive cells, even with low intensity of expression, is associated with poor prognosis too.

- Increased immunoreactivity of NCAD (IRS $\geq 6)$ in primary tumors of cutaneous melanomas is associated with significantly $(p=0.005)$ shorter cancer-specific overall survival. The group of poor prognosis patients is also defined by the parameters of expression of the protein - intense color reaction $(p=0.009)$, and the presence of even only a few NCAD-positive cells $(p=0.024)$.

- Increased immunoexpression of NCAD is positively correlated with more advanced melanomas in Breslow and Clark scale $(p=0.005$ and $p=0.002)$, high mitotic rate $(p=0.036)$ and the presence of lymphangio-invasion ( $p=0.003$ ) and ulceration ( $p=0.002$ for expression intensity).

- Strong expression of NCAD is associated with a more advanced pT $(p=0.009)$, relapse $(p=0.003)$, lymph node metastasis $(p=0.035)$ and distant metastasis $(p=0.008)$.

- SPARC and NCAD have an influence on the risk of death according to Cox univariate analysis $(p=0.0064$ and $p=0.0185$ ), but they are not independent prognostic factors (no significance in Cox multivariate analysis).

\section{References}

Papers of special note have been highlighted as:

- of interest; $\bullet \bullet$ of considerable interest

1 Kalluri R, Weinberg RA. The basics of epithelialmesenchymal transition. J. Clin. Invest. 119(6), 1420-1428 (2009).

-. Reveals basic knowledge about epithelial-mesenchymal transition.

2 Alonso SR, Tracey L, Ortiz P et al. A high-throughput study in melanoma identifies epithelial-mesenchymal transition as a major determinant of metastasis. Cancer Res. 67(7), 3450-3460 (2007).

- Reveals the molecular role of the interplay NCAD and SPARC during epithelial-mesenchymal transition in skin melanoma.

3 Lin K, Baritaki S, Militello L, Malaponte G, Bevelacqua Y, Bonavida $\mathrm{B}$. The role of $B-R A F$ mutations in melanoma and the induction of EMT via dysregulation of the NF- $\kappa \mathrm{B} /$ Snail/ RKIP/PTEN circuit. Genes Cancer 1(5), 409-420 (2010).

4 Taylor MA, Parvani JG, Schiemann WP. The pathophysiology of epithelial-mesenchymal transition induced by transforming growth factor-beta in normal and malignant mammary epithelial cells. J. Mammary Gland Biol. Neoplasia 15(2), 169-190 (2010).

5 Fenouille N, Robert G, Tichet $\mathrm{M}$ et al. The p53/p21 Cip1/Waf1 pathway mediates the effects of SPARC on melanoma cell cycle progression. Pigment Cell Melanoma Res. 24(1), 219-232 (2011).

6 Clark CJ, Sage EH. A prototypic matricellular protein in the tumor microenvironment - where there's SPARC, there's fire. J. Cell Biochem. 104(3), 721-732 (2008).
- Reveals basic knowledge about SPARC.

7 Horie K, Tsuchihara M, Nakatsura T. Silencing of secreted protein acidic and rich in cysteine inhibits the growth of human melanoma cells with G arrest induction. Cancer Sci. 101(4), 913-919 (2010).

8 Redondo P, Lloret P, Idoate M, Inoges S. Expression and serum levels of MMP-2 and MMP-9 during human melanoma progression. Clin. Exp. Dermatol. 30(5), 541-545 (2005).

9 Sage EH, Reed M, Funk SE et al. Cleavage of the matricellular protein SPARC by matrix metalloproteinase 3 produces polypeptides that influence angiogenesis. J. Biol. Chem. 278(39), 37849-37857 (2003).

10 Alvarez MJ, Prada F, Salvatierra E et al. Secreted protein acidic and rich in cysteine produced by human melanoma cells modulates polymorphonuclear leukocyte recruitment and antitumor cytotoxic capacity. Cancer Res. 65(12), 5123-5132 (2005).

11 Sosa MS, Girotti MR, Salvatierra E et al. Proteomic analysis identified N-cadherin, clusterin, and HSP27 as mediators of SPARC (secreted protein, acidic and rich in cysteines) activity in melanoma cells. Proteomics 22, 4123-4134 (2007).

12 Hazan RB, Phillips GR, Qiao RF, Norton L, Aaronson SA. Exogenous expression of $\mathrm{N}$-cadherin in breast cancer cells induces cell migration, invasion and metastasis. J. Cell Biol. 148(4), 779-790 (2000).

13 Giroldi LA, Bringuier PP, Shimazui T, Jansen K, Schalken JA. Changes in cadherin-catenin complexes in the progression of human bladder carcinoma. Int. J. Cancer 82(1), 70-76 (1999). 
14 Mialhe A, Levacher G, Champelovier P et al. Expression of E-, P-, N-cadherins and catenins in human bladder carcinoma cell lines. J. Urol. 164 (3 Pt 1), 826-835 (2000). angiogenesis by regulating monocyte chemoattractant protein-1 expression via PI3K/Akt signaling in prostate cancer cells. Exp. Cell Res. 317(17), 2512-2521 (2011).

W, Lin N, Wang X, Fan QX. Downregulation of $\mathrm{N}$-cadherin expression inhibits invasiveness, arrests cell cycle and induces cell apoptosis in esophageal squamous cell carcinoma. Cancer Invest. 28(5), 479-486 (2010).

17 Chaffer CL, Thompson EW, Williams ED. Mesenchymal to epithelial transition in development and disease. Cells Tissues Organs 185(1-3), 7-19 (2007).

18 Utton MA, Eickholt B, Howell FV, Wallis J, Doherty P. Soluble N-cadherin stimulates fibroblast growth factor receptor dependent neurite outgrowth and $\mathrm{N}$-cadherin and the fibroblast growth factor receptor co-cluster in cells. J. Neurochem. 76(5), 1421-1430 (2001).

19 Williams G, Williams EJ, Doherty P. Dimeric versions of two short Ncadherin binding motifs (HAVDI and INPISG) function as N-cadherin agonists. J. Biol. Chem. 277, 4361-4367 (2002).

20 Kim JB, Islam S, Kim YJ et al. N-cadherin extracellular repeat 4 mediates epithelial to mesenchymal transition and increased motility. J. Cell Biol. 151(6), 1193-1206 (2000).

21 Suyama K, Shapiro I, Guttman M, Hazan RB. A signaling pathway leading to metastasis is controlled by $\mathrm{N}$-cadherin and the FGF receptor. Cancer Cell 2(4), 301-314 (2002).

22 Theisen CS, Wahl JK, Johnson KR, Wheelock MJ. NHERF links the $\mathrm{N}$-cadherin/catenin complex to the platelet-derived growth factor receptor to modulate the actin cytoskeleton and regulate cell motility. Mol. Biol. Cell 18(4), 1220-1232 (2007).

23 Charrasse S, Meriane M, Comunale F, Blangy A, GauthierRouvière C. N-cadherin-dependent cell-cell contact regulates Rho GTPases and beta-catenin localization in mouse $\mathrm{C} 2 \mathrm{C} 12$ myoblasts. J. Cell Biol. 158(5), 953-965 (2002).

24 Navarro P, Ruco L, Dejana E. Differential localization of VE- and N-cadherins in human endothelial cells: VE-cadherin competes with $\mathrm{N}$-cadherin for junctional localization. J. Cell Biol. 140(6), 1475-1484 (1998).

25 Remmele W, Stegner HE. Recommendation for uniform definition of an immunoreactive score (IRS) for immunohistochemical estrogen receptor detection (ER-ICA) in breast cancer tissue. Pathologe 8(3), 138-140 (1987).

Massi D, Franchi A, Borgognoni L, Reali UM, Santucci M. Osteonectin expression correlates with clinical outcome in thin cutaneous malignant melanomas. Hum. Pathol. 30(3), 339-344 (1999).
- As the first, the study reveals the impact of SPARC overexpression for prognosis in skin melanoma patients.

27 Botti G, Scognamiglio G, Marra L et al. SPARC/osteonectin is involved in metastatic process to the lung during melanoma progression. Virchows Arch. 465(3), 331-338 (2014).

28 Ikuta Y, Nakatsura T, Kageshita T et al. Highly sensitive detection of melanoma at an early stage based on the increased serum secreted protein acidic and rich in cysteine and glypican-3 levels. Clin. Cancer Res. 11(22), 8079-8088 (2005).

-. Shows that the concentration of soluble SPARC in the sera of patients with melanomas may be a marker for early-stage melanomas.

29 Robert G, Gaggioli C, Bailet O et al. SPARC represses E-cadherin and induces mesenchymal transition during melanoma development. Cancer Res. 66(15), 7516-7523 (2006).

30 Bachmann IM, Straume O, Puntervoll HE, Kalvenes MB, Akslen LA. Importance of P-cadherin, beta-catenin, and Wnt5a/frizzled for progression of melanocytic tumors and prognosis in cutaneous melanoma. Clin. Cancer Res. 11(24 Pt 1), 8606-8614 (2005).

31 Kreizenbeck GM, Berger AJ, Subtil A, Rimm DL, Gould Rothberg BE. Prognostic significance of cadherinbased adhesion molecules in cutaneous malignant melanoma. Cancer Epidemiol. Biomarkers Prev. 17(4), 949-958 (2008).

- Reveals prognostic significance of NCAD overexpression in skin melanoma.

32 Lade-Keller J, Riber-Hansen R, Guldberg P, Schmidt H, Hamilton-Dutoit SJ, Steiniche T. E- to N-cadherin switch in melanoma is associated with decreased expression of phosphatase and tensin homolog and cancer progression. Br. J. Dermatol. 169 (3), 618-628 (2013).

-• The study of prognostic significance of $\mathrm{E} \downarrow \mathrm{N} \uparrow$ profile.

33 Sandig M, Voura EB, Kalnins VI, Siu CH. Role of cadherins in the transendothelial migration of melanoma cells in culture. Cell Motil. Cytoskeleton 38(4), 351-364 (1997).

34 Li G, Satyamoorthy K, Herlyn M. N-cadherin-mediated intercellular interactions promote survival and migration of melanoma cells. Cancer Res. 61(9), 3819-3825 (2001).

35 Frisch SM, Screaton RA. Anoikis mechanisms. Curr. Opin. Cell Biol. 13(5), 555-562 (2001).

36 Hyeonseok K, Hyeyeon C, Wooseok K et al. Roles of $\mathrm{N}$-kadherin in anoikis and invasion. www.deepdyve.com/pl/elsevier/

-. The role of NCAD in the apoptosis, which is called anoikis. 